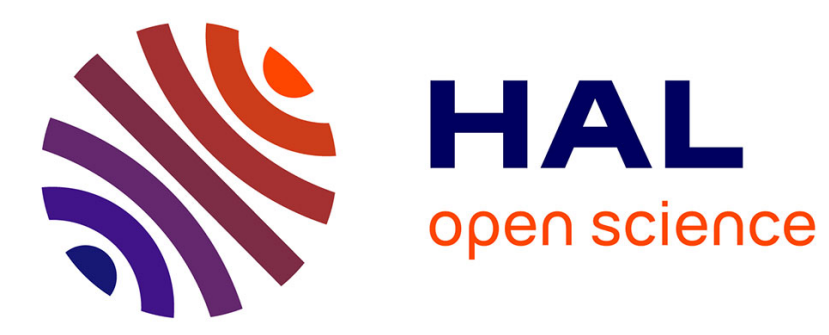

\title{
Vivre ensemble
}

Elena Di Pede

\section{To cite this version:}

Elena Di Pede. Vivre ensemble: quelques pistes bibliques de réflexion. Études Théologiques et Religieuses, 2007, 4 (82), pp.533-548. 10.3917/etr.0824.0533 . hal-02924570

\section{HAL Id: hal-02924570 https://hal.science/hal-02924570}

Submitted on 7 Feb 2022

HAL is a multi-disciplinary open access archive for the deposit and dissemination of scientific research documents, whether they are published or not. The documents may come from teaching and research institutions in France or abroad, or from public or private research centers.
L'archive ouverte pluridisciplinaire HAL, est destinée au dépôt et à la diffusion de documents scientifiques de niveau recherche, publiés ou non, émanant des établissements d'enseignement et de recherche français ou étrangers, des laboratoires publics ou privés. 


\section{VIVRE ENSEMBLE QUELQUES PISTES BIBLIQUES DE RÉFLEXION}

Le "vivre ensemble » est présenté dans la Bible comme un idéal à atteindre (Ps 133), mais aussi, et surtout, comme un lieu où se joue la vie et la mort de l'être humain. À travers un parcours qui va de l'histoire d'Abram à celle de Ruth en passant par la Loi (Ex 20 et Lv 25), Elena DI PEDE* suggère que la Bible, préférant montrer les pièges, les difficultés et les impasses de la vie en société, invite son lecteur à prendre le chemin des relations possibles et vivifiantes pour tous ${ }^{1}$.

Psaume 133

${ }^{1}$ Chant des montées. De David.

Voici ! Qu'il est bon et qu'il est doux

d'être frères aussi ensemble

${ }^{2}$ Comme la bonne huile sur la tête descend sur la barbe,

la barbe d'Aaron, qui descend sur la bouche de ses habits.

${ }^{3}$ Comme la rosée du Hermon, qui descend sur les montagnes de Sion, car c'est là qu'a ordonné le SEIGNEUR la bénédiction,

la vie pour toujours ${ }^{2}$.

Ce court psaume évoque l'idéal du « vivre ensemble », la douceur que la vie vraiment fraternelle procure à ceux qui la vivent. L'image est reposante,

\footnotetext{
* Elena Di PedE est chargé de recherches du FNRS (Fonds national de recherche scientifique) à la Faculté de Théologie de l'Université Catholique de Louvain.

1. Cet article est le texte d'une conférence prononcée lors de la rencontre européenne du MIAMSI (Mouvement International pour l'Apostolat des Milieux Sociaux Indépendants) qui a eu lieu à Bruxelles le 29 avril 2006.

2. Traduction d'après Henri Meschonnic, Gloires. Traduction des Psaumes, Paris, Desclée de Brouwer, 2001. Sur le psaume 133, voir, par ex., Marc GiRARD, Les Psaumes redécouverts. De la structure au sens, vol. II (101-150), Québec, Bellarmin, 1994, p. 382-385.
} 
joyeuse et douce à la fois. La fraternité y semble à toute épreuve, 1'accord est parfait. La réalité décrite est admirable, et le psalmiste le souligne avec émerveillement. Car seule la véritable vie partagée ensemble, en communauté, est une vie pleine de bénédiction, qui peut donc porter des fruits en abondance. L'harmonie est donc complète dans cette communauté, et la mort serait d'être exclu d'une telle beauté et d'un tel bien-être.

Vivre ensemble, c'est bon comme une huile parfumée, comme l'huile précieuse, signe en même temps de l'hospitalité, et donc de la joie qu'il y a à vivre ensemble, et de la consécration au Seigneur, qu'elle soit d'un grand prêtre ou d'un roi. Vivre ensemble a donc quelque chose de sacré dans cet aspect de la vie des humains, mais aussi elle tonifie, comme l'huile qu'utilisent les athlètes avant l'épreuve ; elle est porteuse de guérison, comme l'huile utilisée pour des préparations médicinales ; elle rend beau et protège, comme l'huile utilisé en cosmétique. Vivre ensemble est vivifiant comme la rosée qui abreuve la terre aride : elle est subtile et douce, mais en même temps efficace et féconde, porteuse de fraîcheur et de nouveauté pour ceux qui en bénéficient.

Il m'a semblé important, au début de cet exposé, de mentionner l'idéal, la beauté et le bien-être du « vivre ensemble » tel que le chante le livre des Psaumes, pour camper un décor. Il s'agit là, probablement, d'un but à atteindre. Car il semble bien difficile à rejoindre, comme la Bible elle-même le souligne par ses multiples récits de tentatives malheureuses ou avortées pour connaître cet idéal humain. La Bible, en effet, ne donne pas beaucoup d'exemples réussis du « vivre ensemble » : elle préfère en montrer les pièges, les difficultés, les impasses. D'ailleurs, la toute première expérience biblique du « vivre ensemble » tourne plutôt au cauchemar : souvenons-nous de la famille d'Adam et Ève et des conséquences malheureuses de l'absence de parole entre ses membres, qui aboutit au premier meurtre dans le récit biblique.

Lorsqu'on parle de « vivre ensemble » dans la Bible, on embrasse d'emblée deux dimensions : une dimension horizontale, qui concerne les rapports des humains entre eux, l'autre verticale, qui concerne les rapports des humains avec le Seigneur. Mais on peut affirmer d'emblée que, pour la Bible, l'un ne va pas sans l'autre, et même que, pour que les rapports soient justes entre les humains et le Seigneur, il faut qu'ils le soient avant tout entre les humains eux-mêmes, voire entre les humains et la création entière. Ainsi, pour ouvrir quelques pistes pour réfléchir à la thématique du «vivre ensemble », je vais parcourir des textes tirés du Pentateuque : un récit de la Genèse, un épisode de l'aventure d'Abram, et des lois tirées des livres de l'Exode et du Lévitique. Le premier montrera comment un comportement inadéquat peut bloquer de manière irrémédiable les relations ; le second 
servira à insister sur le fait que pour « vivre ensemble » avec justesse, la loi est indispensable. Enfin, je terminerai par un autre récit, 1'histoire de Ruth, pour montrer comment l'indispensable loi ne va pas sans une certaine inventivité dans la construction du « vivre ensemble ».

Entrons donc sans tarder dans le vif du sujet et lisons le tout début de l'histoire d'Abram dans une traduction plutôt littérale (Gn 11, 26-13, 1) ${ }^{3}$.

$11^{26} \mathrm{Et} \mathrm{Tèrakh} \mathrm{avait} \mathrm{soixante-dix} \mathrm{ans,} \mathrm{et} \mathrm{il} \mathrm{fit} \mathrm{enfanter} \mathrm{Abram} \mathrm{et} \mathrm{Nakhor}$ et Haran. ${ }^{27} \mathrm{Et}$ ceux-ci [sont] les enfantements de Tèrakh. Tèrakh fit enfanter Abram et Nakhor et Haran, mais Haran fit enfanter Lot. ${ }^{28} \mathrm{Et}$ Haran mourut à la face de [a-/de-vant] Tèrakh son père en la terre de son enfantement, en Our-Kasdîm. ${ }^{29}$ Et Abram prit, et Nakhor, pour eux des femmes : nom de la femme d'Abram, Saraï et nom de la femme de Nakhor, Milka fille d'Haran père de Milka et père de Yiska. ${ }^{30} E t$ Saraï fut stérile : il n'y a pas pour elle d'enfant. ${ }^{31} \mathrm{Et}$ Tèrakh prit Abram son fils et Lot fils d'Haran fils de son fils et Saraï sa belle-fille, femme d'Abram son fils, et ils sortirent avec eux de Our-Kasdîm pour aller en terre de Canaan, et ils vinrent jusqu'à Kharan et ils demeurèrent là. ${ }^{32} \mathrm{Et}$ les jours de Tèrakh furent cinq ans et deux-cents ans, et Tèrakh mourut en Kharan.

12'Le Seigneur dit à Abram : «Va-t'en de ta terre et de ton enfantement et de la maison de ton père vers la terre que je te ferai voir, ${ }^{2}$ (pour) que je te fasse en grande nation et que je te bénisse et que je grandisse ton nom et que tu sois bénédiction ${ }^{3} e t$ que je bénisse ceux qui te bénissent - mais qui te méprise, je le maudirai - et qu'en toi acquièrent pour eux la bénédiction tous les clans du sol. " ${ }^{4} \mathrm{Et}$ Abram alla comme lui avait parlé le Seigneur et Lot alla avec lui. Or Abram avait soixante-quinze ans lorsqu'il sortit de Kharan. [...]

Abram parcourt alors le pays du nord au sud, jusqu'au jour où

${ }^{10} \mathrm{Il}$ y eut une famine dans la terre et Abram descendit en Égypte pour résider là car lourde (était) la famine dans la terre. "Et comme il approchait pour entrer en Égypte, il dit à Saraï sa femme : "Voici je te prie, je sais que tu es une femme belle de vue. ${ }^{12}$ Lorsque les Égyptiens te verront, ils diront: "c'est sa femme" et ils tueront moi, mais toi ils feront vivre. ${ }^{13}$ Dis je te prie (que) tu es ma sœur afin que ce soit bien pour moi grâce à [par le revenu de] toi et que vive mon être à cause [dans le retournement] de toi. » ${ }^{14} \mathrm{Et}$ quand Abram entra en Égypte, les

3. Sur ce récit, on lira avec profit André WéNıN, «Abram, fils de Tèrakh. Une interprétation de Genèse 11,'26-32 », Cahiers de l'École des Sciences philosophiques et religieuses 20, 1996, p. 135-151 et, du même auteur; «Abram et Saraï en Égypte (Gn 12,10-20) ou la place de Saraï dans l'élection », Revue théologique de Louvain 29, 1998, p. 433-456. 
Égyptiens virent la femme, qu'elle était très belle. ${ }^{15} \mathrm{Et}$ la virent des princes [hébr. : sarei] de Pharaon, et ils la louèrent vers Pharaon et la femme fut prise (dans la) maison de Pharaon. ${ }^{16} \mathrm{Et}$ à Abram, il fit du bien grâce à [par le revenu d'] elle, et il y eut pour lui petit et gros bétail et ânes et serviteurs et domestiques et ânesses et chameaux.

${ }^{17}$ Et le Seigneur frappa Pharaon de grandes frappes ainsi que sa maison, à cause [sur le propos] de Saraï, la femme d'Abram. ${ }^{18}$ Et Pharaon appela Abram et dit : "Que m'as-tu fait là ? Pourquoi ne m'as-tu pas informé qu'elle est ta femme? ${ }^{19}$ Pourquoi as-tu dit : "Elle est ma sceur" - et je me la suis prise pour femme? Et maintenant, voici ta femme! Prends et va! » ${ }^{20} \mathrm{Et}$ Pharaon ordonna sur lui des hommes et ils (le) raccompagnèrent/laissèrent aller lui et sa femme et tout ce qui (était) à lui. 13'Et Abram monta d'Égypte lui et sa femme et tout ce qui est à lui et Lot avec lui vers le Néguev ${ }^{4}$.

Nous sommes au début de l'histoire d'Abram, et cet épisode montre combien il est difficile de vivre ensemble, surtout avec des étrangers que l'on ne connaît pas, mais aussi à l'intérieur d'un couple, formé ici par Abram et Saraï. Mais voyons dans le détail ce qui se passe dans ce petit récit.

Après le dramatique récit du déluge et l'aventure de Babel, là où l'humanité cherche l'unité par l'uniformité, ce qui ne plaît pas au Seigneur, le récit se centre sur la famille de Tèrakh, le père d'Abram (Gn 11, 26-32). Tel qu'il est dépeint, ce portrait de famille donne une image assez particulière du père, Tèrakh. C'est quelqu'un qui domine et veut tout contrôler dans sa famille, dont il semble disposer comme il le veut, «faisant enfanter pour lui » et " prenant» les siens qui semblent ne pas avoir grand-chose à dire. De plus, dans cette famille, tous les liens se tissent à l'intérieur du clan et les membres se marient entre eux, à l'exception d'Abram. Car Saraï n'est pas issue de ce clan, à la différence de Milka qui est la nièce de son mari Nakhor. Il s'agit là d'un élément important sur lequel il faudra revenir.

Après ce petit aperçu général sur la famille, le narrateur se focalise sur l'histoire d'un de ses membres, Abram, celui qui s'était déjà singularisé à l'intérieur du clan par son mariage avec une femme qui n'en est pas issue ${ }^{5}$. L'aventure d'Abram commence par un appel du Seigneur qui l'invite à partir loin de cette famille, pour aller vers le pays que le Seigneur lui montrera. Le but affiché de ce voyage est la bénédiction pour tous ceux qui béniront Abram. Ainsi, Abram est élu en vue de la vie et du bonheur pour tous. Mais pour que tous aient la vie et la bénédiction, il faut que l'élu en question

4. Traduction d'après A. WéNIN. Les italiques sont du traducteur ; elles mettent en évidence les dialogues entre les personnages. Entre crochets, nous proposons une traduction en évidence issue du clan. 
occupe sa place propre parmi les autres de manière juste, ce qui semble suggérer que, pour vivre ensemble avec ceux qu'il rencontre, Abram doit occuper une place particulière, une place qu'il doit peut-être trouver aussi grâce aux autres.

La parole adressée à Abram par le Seigneur - l'ordre de quitter la maison de son père pour aller là où le Seigneur lui montrera - est au fond une invitation à la confiance, car c'est la parole d'un autre qui le pousse au-delà du désir de son père, et donc loin de la sécurité du nid familial. En effet, lorsqu'il se met en voyage de Our des Chaldéens pour aller en Canaan, Tèrakh s'arrête à mi-chemin, dans un lieu nommé Kharan, un lieu dont le nom rappelle étrangement, par sa sonorité, le nom de Haran, le fils mort de Tèrakh $(11,28)$. Il semblerait donc que le voyage du clan s'arrête, se fige dans le passé, dans le souvenir du fils qui n'est plus. Mais Abram est appelé à reprendre la route, à se mettre en route vers le futur, un futur dont il ne sait rien, mais le Seigneur lui dit qu'il sera positif et qu'il comporte une mission pour lui : amener la bénédiction, et donc la vie du Seigneur, à tous les clans de la terre s'ils savent reconnaître en lui le messager de cette vie partagée. Telle semble être la condition de la vie ensemble : pour l'élu - Abram -, reconnaître l'autre, ses spécificités et ses richesses ; pour l'autre - le non-élu -, reconnaître Abram et ce qu'il est. Ainsi la reconnaissance mutuelle des différences légitimes fera en sorte que la vie circule pour tous et entre tous. Mais les choses ne seront pas aussi simples que l'on pourrait croire, et le premier à avoir du mal avec sa mission sera Abram lui-même, on va le voir.

Abram prend donc la route avec sa maisonnée, reprenant le voyage que son père avait interrompu. Il se met en marche sans opposer aucune objection à l'ordre du Seigneur, alors qu'il aurait pu avoir des raisons objectives de refuser l'aventure : son grand âge, par exemple, ou la stérilité de sa femme, deux obstacles à la grande descendance promise par Dieu (v. 2). Puis, une fois arrivé là où Tèrakh avait initialement prévu d'arriver, le Seigneur parle à nouveau à Abram (v. 7) et lui fait voir le lieu où il pourra s'installer. Abram répond en construisant deux autels pour le Seigneur et en invoquant son nom. Mais Abram va bientôt devoir apprendre à gérer son statut particulier, non plus dans un rapport à deux (lui et le Seigneur), mais dans le rapport à trois, c'est-à-dire en interaction avec d'autres qui ne font pas partie de son clan. La suite du récit montre combien cela est difficile.

À l'occasion d'une famine, Abram décide de descendre en Égypte pour y résider. Il s'agit ici de la première rencontre entre le patriarche et ces étrangers. Abram ne les connaît donc pas directement. De plus, le narrateur n'a jamais fait mention du fait que quelqu'un aurait rapporté des us et coutumes égyptiens à Abram ou à quelqu'un des siens. Pourtant, Abram se comporte comme s'il les connaissait. Mais les connaît-il vraiment ? Quoi qu'il en soit, 
il s'adresse à sa femme et lui dit qu'il sait qu'elle est belle et que cette beauté va attiser la convoitise des Égyptiens, qui vont certainement devenir agressifs, le tuant pour lui prendre sa femme. C'est la raison pour laquelle il supplie Saraï de dire qu'elle est sa sœur, afin que les méchants étrangers l'épargnent, et qu'au contraire lui s'enrichisse grâce à ce subterfuge. Abram a peur. C'est clair. Mais cette peur est-elle fondée ? Abram n'est-il pas en train de fantasmer autour de ce qu'il ignore ? Car le lecteur peut raisonnablement se demander sur quoi il fonde cette supplique adressée à Saraï. Aucun élément du récit n'appuie le point de vue du patriarche qui entraîne sa femme dans sa logique, la priant de mentir pour lui. On s'en souvient, en effet : en décrivant la famille de Tèrakh, famille pétrie de liens internes, le narrateur n'a fait aucune allusion au fait que Saraï est la sœur d'Abram.

Rien qu'en lisant ces paroles, le lecteur peut déjà imaginer que l'aventure d'Abram commence mal. Car il va à la rencontre de l'autre rempli de peurs imaginaires à son sujet et de désirs pour lui-même. Ce faisant, il risque de lui barrer la route de la bénédiction dont il est porteur afin que tous puissent vivre, lui et les autres (y compris les étrangers). Il faut remarquer que, dans cet épisode, le Seigneur n'intervient pas, laissant à Abram lui-même le soin de se mesurer avec sa mission et de s'ajuster à elle.

Une fois en Égypte, les choses se passent exactement comme Abram l'avait imaginé. Les Égyptiens trouvent effectivement que Saraï est très belle, la font entrer dans le harem de Pharaon, et enrichissent effectivement Abram comme il l'avait souhaité. Abram avait-il donc raison lorsqu'il imaginait les Égyptiens comme des prédateurs ? N'est-ce pas plutôt son attitude et le scénario qu'il a imaginé avant la rencontre qui permettent que les choses prennent cette tournure ? Saraï est effectivement une très belle femme, le narrateur le dit. Rien d'étonnant, donc, que ces étrangers soient attirés par elle. Mais elle n'est pas seulement belle : elle est également libre aux yeux des Égyptiens qui la croient sœur d'Abram. Les choses ne se seraient pas passées de cette manière si Abram avait été honnête avec ces hommes dès le début. Mais pour cela, il aurait dû être ouvert à une rencontre authentique, en prendre le risque sans avoir peur. Rien dans le texte, en effet, ne permet de dire que les événements se seraient déroulés comme Abram les a imaginés. Car ni ici, ni dans ce qui précède, le narrateur, je le répète, ne dit rien d'une éventuelle méchanceté des Égyptiens ou de Pharaon.

Ainsi, le mensonge d'Abram ne respecte ni sa femme (qui se laisse faire pendant un temps) ni ses interlocuteurs. Il ne respecte pas non plus la mission que le Seigneur lui a confiée et barre de ce fait le chemin de la bénédiction. C'est donc l'élu qui dans le cas présent est responsable de la tournure dramatique que prendront les événements. Car en ne disant pas la vérité, Abram pousse en quelque sorte Pharaon à enfreindre une loi à son insu, à outrepas- 
ser une limite qu'il n'aurait peut-être pas dépassée autrement. Mais la suite est éloquente : l'attitude d'Abram pousse les Égyptiens vers la mort. Le Seigneur lui avait dit que vivraient ceux qui le reconnaîtraient et que seraient maudits ceux qui ne le reconnaîtraient pas. C'est effectivement ce qui se passe : Abram n'ayant pas permis aux Égyptiens de se situer clairement face à lui, il les a poussés à la faute et le Seigneur les frappe. Par son agir même, Abram est porteur de mort chez ses hôtes, alors que sa mission l'appelait à être porteur de vie par la bénédiction. Ainsi frappé, Pharaon semble prendre conscience de la relation injuste qu'il entretient avec la femme d'un autre. Il décide donc d'y mettre un terme. Du coup, il rétablit la justesse et la justice dans le couple Abram/Saraï. Il lui donne ainsi une belle leçon de vie : s'il ne respecte ni sa femme ni l'étranger en face de lui, il lui sera impossible de « vivre ensemble », voire même de vivre tout simplement. Et il faut remarquer que c'est l'étranger, celui dont Abram avait peur, qui lui permet de sortir du mensonge dans lequel il s'était enfermé.

Cette petite histoire montre bien que, même si le Seigneur reste aux côtés d'Abram envers et contre tout, la vie ne peut circuler que si tous les acteurs humains respectent l'autre, chacun à sa place, sans jalousie, sans convoitise et aussi, c'est essentiel, sans peur. Dans le cas contraire, c'est la mort pour tous : pour les Égyptiens qui sont frappés, mais aussi, probablement pour Abram lui-même qui se coupe de sa relation avec son épouse et avec les autres.

Vivre ensemble n'est donc pas une mince affaire, que ce soit avec des étrangers, comme nous venons de le voir, mais aussi, c'est clair, avec ceux qui nous ressemblent parce qu'ils font partie de la même famille, communauté, groupe social, etc. C'est probablement la raison pour laquelle des lois sont nécessaires pour régir la vie commune. Ces lois sont nombreuses dans le Pentateuque, et réglementent la vie communautaire dans tous ses aspects : entre le peuple et son Seigneur, entre les membres du peuple, vis-à-vis des faibles - la veuve, l'orphelin, les pauvres et l'étranger. Pour illustrer comment la Loi donne un cadre pour vivre ensemble, nous allons nous pencher sur deux passages : l'un bien connu, la fin du décalogue, l'autre qui l'est probablement un peu moins, le chapitre 25 du Lévitique.

Lisons tout d'abord dans les Dix Paroles, dans la version qu'en donne le chapitre 20 du livre de l'Exode, ce qui concerne directement notre sujet ${ }^{6}$.

6. Pour aller plus loin sur les Dix Paroles : Paul BeAuchamp, « Au cœur de la Loi, le Décalogue », Croire aujourd'hui 184, 1987, p. 259-270 ; D'une montagne à l'autre. La Loi de Dieu, Paris, Seuil, 1999 ; « La loi du décalogue et l'image de Dieu », Croire aujourd'hui 186, 1987, p. 397-406 ; André WéNIn, L'homme biblique. Lectures dans le premier Testament, Paris, Cerf, coll. «Théologies », 2004², p. 109-133. 
${ }^{8} \mathrm{Se}$ souvenir du jour du sabbat pour le sanctifier. ${ }^{9} \mathrm{Six}$ jours, tu serviras et tu feras tout ton ouvrage ; ${ }^{10}$ mais le septième jour est sabbat pour le SEIGNEUR ton Élohîm : tu ne feras aucun ouvrage, toi et ton fils et ta fille, et ton serviteur et ta domestique et ton bétail et ton étranger qui est dans tes portes. " $\mathrm{Car}$ six jours le SeIGneur a fait le ciel et la terre et la mer et tout ce qui est en eux et il s'est reposé au septième jour. ${ }^{12}$ Honore ton père et ta mère, afin que se prolongent tes jours sur le sol que le SEIGNEUR ton Élohîm va te donner. ${ }^{13} \mathrm{Tu}$ ne tueras pas ${ }^{14} \mathrm{et}$ tu n'adultèreras pas ${ }^{15} \mathrm{et}$ tu ne voleras pas ${ }^{16} \mathrm{et}$ tu ne témoigneras pas contre ton prochain un témoignage d'imposture ${ }^{17} \mathrm{et}$ tu ne convoiteras pas la maison de ton prochain et tu ne désireras pas la femme de ton prochain, et son serviteur et sa domestique, et son bœuf et son âne et tout ce qui est à ton prochain ${ }^{7}$.

Après une première partie où il s'agit de la relation juste avec le Seigneur, à partir du centre commencent les règles du bon vivre en société. Le premier précepte en ce sens, c'est le sabbat. En le respectant, l'humain imite son créateur, fait du temps de Dieu, qui s'est reposé au septième jour pour laisser place à la création, son propre temps, un temps où il choisit de laisser place à l'autre : sa famille, ses bêtes et les étrangers qui, eux aussi, ont droit au repos après le travail accompli. Ainsi, le jour du sabbat, l'homme accepte de suspendre le travail pour ne pas en être esclave et faire place à d'autres valeurs, relationnelles celles-là.

Après avoir invité l'homme à couler ses rythmes dans ceux de Dieu, comme pour suggérer que se joue là quelque chose d'important pour l'alliance, le décalogue énumère des préceptes qui concernent spécifiquement le « vivre ensemble ». Tout d'abord, il s'agit de vivre avec ceux qui nous précèdent : les parents. Les respecter est un moyen de prolonger ses propres jours, c'est-à-dire de vivre sur la terre que le Seigneur a donnée au peuple. Respecter les parents signifie, en effet, accueillir positivement ce qu'ils nous transmettent : la vie et la terre, mais aussi la Loi, le mode d'emploi du bien-vivre dans cette terre donnée par le Seigneur. Les derniers préceptes sont tous négatifs. Ils forment la base de la vie en société. Pour que cette vie en société soit possible, il y a des choses à ne pas faire : tuer, commettre l'adultère et voler. Ces trois actions non seulement privent l'autre de quelque chose de précieux qui lui appartient mais, en plus, elles introduisent la violence dans la relation qui, par le fait même, se brise. Car la violence engendre la violence et plus aucune relation authentique n'est possible lorsqu'elle est présente. Ainsi, pour tenter de l'enrayer dès son point de départ, pour tenter de la tuer dans l'œuf, pour ainsi dire, le décalogue

7. Traduction d'après A. Wénin. 
prévient cette violence non seulement dans les actes, mais aussi dans les intentions. Pour cela, il invite à ne pas témoigner par un mensonge contre le prochain, car dire le faux sur quelqu'un risque d'entraîner sa mort, cette fois non pas biologique mais sa mort du point de vue des relations sociales. Le décalogue invite également à ne pas convoiter les biens et la femme du prochain. La convoitise, en effet, est cette disposition intérieure qui ouvre précisément la porte à la violence. Car celui qui convoite ne se réjouit pas pour le bien de l'autre, mais souhaite se l'approprier injustement. Ainsi, ce n'est pas seulement dans les faits qu'il faut refuser la violence pour que le « vivre ensemble » soit possible. Il faut également la refuser dans les intentions, montrant que l'on rejette toute jalousie et toute convoitise par rapport à l'autre et à ce qu'il est. Car refuser le mal de manière parfois un peu abstraite ne suffit pas, il faut aussi refuser d'en emprunter le chemin.

Le décalogue c'est la base, le minimum. Sans respect de ces préceptes de base, aucun « vivre ensemble » n'est possible. Mais une fois cette base assurée, il y a des manières d'être à développer, une mentalité à développer. En ce sens, on peut lire, par exemple, Lv 25. Il s'agit de l'année jubilaire, une année durant laquelle toutes les pendules sont remises à l'heure pour permettre à la terre de se reposer et aux plus pauvres, qui auraient des dettes, de prendre un nouveau départ. Lisons donc ensemble les parties de ce texte significatives pour notre propos et voyons comment les rapports entre humains mais aussi entre les humains et la création - ici symbolisée par la terre $-\mathrm{y}$ sont réglés en vue d'un « vivre ensemble » porteur de vie. Arrêtonsnous sur quelques lignes significatives de ce long chapitre ${ }^{8}$.

Lévitique 25

${ }^{1}$ Et le Seigneur parla à Moïse au mont Sinaï disant : ${ }^{2}{ }^{2}$ Parle aux fils d'Israël et tu leur diras : "Quand vous viendrez vers la terre que je vous donne, la terre fera sabbat un sabbat pour le SEIGNEUR. ${ }^{3} \mathrm{Six}$ années tu ensemenceras ton champ et six années tu tailleras ta vigne ; et tu amasseras sa récolte. ${ }^{4} \mathrm{Mais}$ à la septième année, un sabbat sabbatique sera pour la terre, un sabbat pour le SEIGNEUR ; ton champ, tu ne l'ensemenceras pas, et ta vigne, tu ne la tailleras pas ; 'Sle regain de ta moisson tu ne moissonneras pas et les raisins de tes vignes non taillées tu ne les vendangeras pas, une année sabbatique sera pour la terre. ${ }^{6} \mathrm{Et}$

8. La traduction du chapitre 25 du Lévitique suit celle de Didier Luciani, Sainteté et pardon, vol. II. Guide technique, Louvain, Peeters, coll. « BETL 185 B », p. 473-476. Sur ce chapitre, voir également Jean-François LeFEBVRE, Un mémorial de la création et de la rédemption. Le jubilé biblique en Lv 25, Bruxelles, Lumen Vitae, coll. " Connaître la Bible 23 ", 2001 ; Didier LUCIANI, " Le jubilé dans Lévitique 25 », Revue théologique de Louvain 30, 1999, p. 456-486 ; Jean-Marie VAN CANGH, « Le jubilé biblique : un temps marqué ouvrant un temps neuf », Science et Esprit 53, 2001, p. 63-92 ; André WÉnIN, Le sabbat dans la Bible, Bruxelles, Lumen Vitae, coll. « Connaître la Bible 38 », 2005, p. 52-54. 
On le voit, à côté du cadre temporel, les diverses modalités qui régulent le recouvrement de la liberté et de la propriété mettent en place un cadre social. Ce cadre doit s'accompagner, soulignent les versets 14 et 17, d'honnêteté dans les transactions :

${ }^{14} \mathrm{Et}$ quand vous faites du commerce - que tu vendes quelque chose à ton compatriote, ou que tu achètes quelque chose de lui -, que nul d'entre vous n'exploite son frère. [... ${ }^{17} E$ Et vous n'abuserez pas chacun son compatriote, mais tu craindras ton Dieu, car je suis le SEIGNEur, votre Dieu.

En respectant les règles ainsi établies, l'humain peut vraiment faire place à l'autre en face de lui : la terre, les autres humains, mais aussi le Créateur dont lui vient la terre et la Loi nécessaire pour y vivre l'alliance. Ainsi, le temps d'arrêt dans l'exploitation de la nature et des esclaves devient un moyen important de cohésion pour le peuple dans la mesure où il corrige les inégalités tant sociales qu'économiques qui voient inévitablement le jour lorsque la loi du plus fort domine les relations. Aussi, semble dire cette première partie du chapitre, le rapport ajusté au temps donne au peuple la possibilité de repartir régulièrement sur de nouvelles bases, effaçant l'ardoise pendante dans la relation à Dieu et dans les relations humaines.

Si l'Israélite se comporte de cette manière, il vivra en sécurité sur la terre (v. 18) et mangera à satiété, même pendant la septième année, car le Seigneur enverra sa bénédiction afin que la sixième année, la récolte soit abondante au point de couvrir les besoins de trois ans, jusqu'à la nouvelle récolte après l'année sabbatique (v. 19-24). Comme pendant l'exode, le peuple est invité à faire confiance à son Dieu, qui promet la nourriture. Au désert, il s'agissait de la manne. Ici c'est la terre qui produira abondamment en prévision de la jachère. Celle-ci devient dès lors comme un test qui montre comment le peuple se conforme à la loi. Car ici la nourriture ne tombe plus du ciel, comme cela arrivait avec la manne, mais elle pousse du sol. Dans ce cas, les règles doivent être claires, pour éviter que celui qui cultive cette terre ne soit tenté de l'exploiter sans limites, sans lui laisser de répit. S'il lui concède du repos, c'est qu'il consent au manque, à la non-mainmise. C'est qu'il accepte de ne pas tout avoir. Pour le dire autrement, il refuse la convoitise. C'est ainsi qu'il ouvre un espace de vie dans lequel l'autre pourra trouver sa place. Dans le cas contraire, vivre et vivre ensemble est impossible. La gestion de la terre devient donc le nœud où s'articulent les relations à l'autre et au Seigneur. En effet, s'il entre dans une démarche de refus de la convoitise par rapport à la terre, l'humain sera aussi à même d'avoir un même état d'esprit en ce qui concerne sa relation à l'autre et aux autres. La loi invite à l'imitation de l'agir divin qui doit être un modèle et une norme pour l'agir des Israélites. Cette manière d'agir passe inévitablement par la fidélité à la Loi, par laquelle le 
peuple est appelé à la confiance envers le Seigneur et à une solidarité réelle et effective envers autrui.

Ensuite le chapitre 25 explicite la règle du go' $e l$, un membre de la famille qui a le devoir de racheter le bien d'un parent dans la détresse financière :

${ }^{25}$ Quand ton frère sera dans la gêne et vendra de sa propriété, alors viendra vers lui son racheteur $\left(g o^{\prime} e l\right)$, le [plus] proche et il rachètera la vente de son frère.

Mais tous n'ont pas la chance d'avoir un proche, un go'el. Ainsi, la suite prévoit les modalités pour que quelqu'un qui serait dépourvu de go'el puisse racheter son bien par lui-même, lors de l'année jubilaire, avec de l'argent ou par son travail (v. 26-34). Ainsi, la loi prévient le risque de dispersion de l'espace familial et de son patrimoine, mis en danger par la vente d'une parcelle ou d'une maison. Elle prévient par le fait même le risque de concentration de la richesse dans la main de quelques personnes de plus en plus riches.

Après avoir parlé du rachat des biens, et avant de se tourner vers le rachat des membres du peuple qui se seraient vendus comme esclaves pour payer leurs dettes, Lv 25 explicite quelques règles pour que les rapports entre les humains soient le plus juste possible (v. 35-38) :

${ }^{35} \mathrm{Et}$ quand ton frère sera dans la gêne et que sa main vacille près de toi, alors tu le soutiendras immigré et résident, et il vivra près de toi. ${ }^{36} \mathrm{Ne}$ prends de lui ni intérêt, ni usure, mais tu craindras ton Dieu et ton frère vivra près de toi. ${ }^{37}$ Ton argent, tu ne lui donneras pas à intérêt, et à usure tu ne lui donneras pas ta mangeaille. ${ }^{38} \mathrm{Je}$ suis le SEIGNEUR votre Dieu qui vous ai fait sortir de la terre d'Égypte pour vous donner la terre de Canaan pour être pour vous Dieu.

L'Israélite est invité à soutenir ses proches, et cela en n'exigeant pas de lui des intérêts. On le voit : la loi protège les économiquement faibles contre les abus de pouvoir dont les riches pourraient faire preuve. À l'image de Dieu, en effet, le plus fort doit être libérateur de son partenaire et non la source de ses difficulté ou, pire, son esclavagiste. Après avoir ainsi souligné le cadre général, le législateur peut se centrer sur le rachat des personnes qui, malgré tout, auraient dû se vendre elles-mêmes pour payer leurs dettes (v. 3945) :

${ }^{39} \mathrm{Et}$ quand ton frère près de toi sera dans la gêne et il sera vendu à toi, tu ne l'asserviras pas à un service de serviteur. ${ }^{40}$ Comme un journalier, comme un résident il sera près de toi, jusqu'à l'année du jubilé il servira près de toi. ${ }^{41}$ Et il sortira d'auprès de toi, lui et ses fils avec lui, et il retournera vers sa famille ; vers la propriété de ses pères il retournera. ${ }^{42}$ Car mes serviteurs, eux que j'ai fait sortir de la terre d'Égypte, 
ils ne seront pas vendus comme on vend des esclaves. ${ }^{43} \mathrm{Tu}$ ne domineras pas sur lui avec rigueur, mais tu craindras ton Dieu.

L'homme qui s'est vendu ne doit pas être traité comme un serviteur, comme un esclave. Au contraire, ce serait prendre le risque de le déshumaniser et probablement aussi de se déshumaniser. Aussi, le patron doit se montrer plein d'humanité envers lui. Encore une fois - mais est-ce étonnant ? - le précepte prend comme modèle l'agir salvifique et libérateur du Seigneur qui a libéré son peuple de l'esclavage égyptien. Le riche consentira-t-il à ne pas devenir pharaon pour ses frères ? Imitera-t-il l'agir de Dieu pour que s'établissent des rapport sociaux justes entre tous ? Tel est l'enjeu fondamental de cette loi qui promeut, par des modalités précises, l'égalité entre tous.

Un israélite dans la détresse n'a pas seulement la possibilité de vendre ses biens ou de se vendre lui-même à l'un des membres de son peuple. Il peut également se vendre à un étranger. Une fois encore, la loi prévoit les modalités pour qu'il puisse retrouver sa liberté et prendre un nouveau départ (v. 4755). Car ici, l'Israélite ne risque pas seulement de perdre sa liberté ; il risque aussi de perdre son identité, absorbé qu'il est par une famille étrangère. Il est donc fondamental de ne pas l'abandonner à son sort. Encore une fois, c'est son proche parent qui pourra le racheter lors de l'année jubilaire selon des modalités bien précises.

Ce qui est central dans toute cette législation, c'est la solidarité avec la création, entre les membres d'une même famille, d'un même clan, mais aussi avec l'étranger. De cette manière, la loi met en garde et cherche à freiner les conséquences néfastes d'une manière d'agir où quelqu'un oublierait le don de Dieu et asservirait la terre et les autres à son profit exclusif. Et bien plus qu'une simple action qui ne serait que justice, cette solidarité est calquée sur l'imitation de l'agir divin, un agir libérateur qui permet la liberté et donc la vie de son peuple.

Cette loi qui invite le peuple à faire mémoire très concrètement, jour après jour, de la libération, est garantie par le Seigneur lui-même qui affirme qu'il entendra le cri de la veuve et de l'orphelin maltraités. Cette loi dont le Seigneur est la source, le fondement et la garantie, est donc nécessaire au bon fonctionnement de la société.

Mais la loi, bien qu'elle soit un élément capital du « vivre ensemble » n'est pas le seul ingrédient nécessaire. L'histoire de Ruth l'illustre abondamment ${ }^{9}$.

9. Pour approfondir la lecture du livre de Ruth, voir, par ex., Aldina DA SiLVA, Ruth. Un évangile pour la femme aujourd'hui, Montréal, Mediaspaul, coll. " Parole d'actualité 4 », 1996 ; Carlos Mesters, Ruth. L'amour engendre la justice, Bruxelles, Lumen Vitae, coll. "Connaître la Bible 34 », 2004 ; André WÉNIN, Le livre de Ruth. Une approche narrative, Paris, Cerf, coll. « Cahiers Évangile 104 », 1998. 
Le récit commence alors qu'une famine (encore une) sévit dans le pays $(1,1)$. C'est la raison pour laquelle un homme de Bethléem quitte son village natal pour se rendre au pays de Moab, en Transjordanie, avec sa femme Noémi et ses fils. Après la mort de leur père, ceux-ci épousent des moabites, Orpa et Ruth (1,3-4). Mais voilà que, après une dizaine d'années, les deux fils de Noémi meurent à leur tour laissant veuves les trois femmes $(1,5)$. C'est alors que Noémi entend dire que la famine est finie. Elle décide de retourner dans son pays, en Juda (1, 6-7). Mais elle ne veut pas forcer ses brus à la suivre, leur rendant leur liberté pour qu'elles restent chez elles $(1,8$ 13). Puis $\left(1,14-18^{10}\right)$.

${ }^{14}$ Et elles levèrent leur voix et pleurèrent encore et Orpa embrassa (une dernière fois) sa belle-mère, mais Ruth s'attacha à elle. ${ }^{15} \mathrm{Et}$ elle dit : «Voici que ta belle-sæur est retournée vers son peuple et vers son Dieu [ses dieux]. Retourne derrière ta belle-sœur. " ${ }^{16} \mathrm{Et}$ Ruth dit : «N'insiste pas auprès de moi pour t'abandonner en retournant de derrière toi car où tu iras, j'irai et où tu logeras, je logerai ; ton peuple (sera) mon peuple et ton Dieu sera mon Dieu; ${ }^{17}$ où tu mourras, je mourrai, et là je serai enterrée. Ainsi fera le SEIGNEUR pour moi et ainsi ajoutera! Oui, c'est la mort qui nous séparera moi et toi. ${ }^{18} \mathrm{Et}$ Noémi vit que Ruth était résolue à parler avec elle et elle cessa de lui parler.

Ruth est têtue, refusant de laisser sa belle-mère, contrairement à ce que fait Orpa. Mais dans ses paroles on peut aussi sentir sa détermination, son affection, sa solidarité avec une femme malheureuse et aussi, pourquoi pas, une certaine confiance de Ruth, en tout cas par rapport au Seigneur. Détermination, affection, solidarité et confiance, quelques ingrédients utiles lorsqu'il s'agit de « vivre ensemble».

Les deux femmes se mettent donc en route et arrivent au village. Les femmes les accueillent, mais Noémi leur dit combien elle est amère à cause du sort que le Seigneur lui a fait subir $(1,19-22)$, comme si elle se sentait plongée dans la mort sans voir aucune issue. Nous sommes à la période des moissons et, pour assurer sa survie ainsi que celle de sa belle-mère, Ruth part glaner dans les champs $(2,2-3)$. La loi protège en effet les pauvres qui glanent, c'est-à-dire qui passent derrière les moissonneurs et ramassent quelques épis oubliés afin d'assurer leur quotidien. Cette loi prévoit même que les moissonneurs laissent volontairement quelques épis à l'intention des pauvres. Arrivant à son champ, Boaz, un parent du défunt mari de Noémi, voit la jeune fille et s'inquiète de savoir qui elle est. Apprenant qu'elle est la

10. Traduction d'après André WéNIN, ibid., p. 33-36. Pour l'usage des italiques et des crochets, voir note 4 . 
belle-fille de Noémi, il la prend explicitement sous sa protection, manière pour lui de la remercier pour tout ce qu'elle a fait pour sa parente, Noémi, après la mort de son mari $(2,4-23)$. Constatant cette protection affichée de Boaz pour Ruth, qui lui a permis de ramasser beaucoup d'orge (v. 17-19), Noémi se souvient qu'il est leur go'el (v. 20). Une idée lui vient alors.

\section{Rt 3}

'Et Noémi sa belle-mère lui dit: «Ma fille, ne chercherais-je pas pour toi un repos qui sera bon pour toi ? ${ }^{2}$ Et maintenant, n'est-il pas notre parent, ce Boaz avec les jeunes filles de qui tu as été ? Voici : il vanne l'air des orges la nuit. ${ }^{3}$ Tu te laveras, tu te parfumeras, tu mettras ton manteau sur toi et tu descendras à l'aire. Ne sois pas connue de l'homme jusqu'à ce qu'il ait achevé de manger et de boire. ${ }^{4} E t$ quand il se couchera, tu connaîtras le lieu où il se couchera, et tu viendras et tu te découvriras à l'endroit de ses pieds et tu te coucheras, et lui, il te racontera ce que tu devras faire. »

Apparemment, Noémi cherche une manière de forcer l'impasse de la mort dans laquelle Ruth et elle se trouvent. Boaz est leur go'el, il a le devoir de ne pas les laisser dans le dénuement, il a le devoir de sortir définitivement ces deux femmes du dénuement. Car selon la loi du lévirat, un frère (et en absence de celui-ci, un proche parent) est dans l'obligation de donner une descendance à son frère défunt en épousant sa veuve pour que celui-ci ait une postérité. C'est sans doute ce que Noémi a en vue quand elle fait preuve d'initiative et d'inventivité, en envoyant sa bru se coucher aux pieds de Boaz. Pour Ruth, cela implique une prise de risque : comment l'homme réagira-t-il, en effet ? Ainsi, initiative, inventivité et prise de risque s'ajoutent à la détermination, à l'affection, à la solidarité et à la confiance. Peut-être même que ces trois nouveaux ingrédients découlent des autres.

Découvrant la femme à ses pieds, Boaz est certainement étonné $(3,7-8)$. Pourtant il accepte immédiatement son rôle de go'el. Mais un problème subsiste car, dit-il, les deux femmes ont un parent encore plus proche que lui. Puis, laissant Ruth passer la nuit avec lui, il lui assure qu'il exercera son devoir si l'autre parent ne manifeste pas l'intention de le faire $(3,12-13)$. Dans tout cela, Boaz reconnaît la présence agissante du Seigneur, mais aussi la sagesse, et le sens des responsabilités et du devoir de la jeune fille, qu'il reconnaît comme une femme de valeur. En réalité, le proche parent auquel Boaz faisait allusion n'exercera pas son droit (4, 1-8). En effet, Boaz lui présente les choses de telle sorte qu'elles apparaissent peu intéressantes pour lui et qu'il préfère renoncer. Boaz peut alors faire ce qu'il a promis à Ruth : il rachète une terre de Noémi $(4,9-12)$ et prend Ruth comme femme $(4,13)$. De cette union naîtra un fils, dont la naissance fait revivre aussi Noémi (4, 14-17). Ainsi, non seulement la loi est suivie, mais elle suscite l'inventi- 
vité de Boaz qui semble vraiment disposé à aider les deux femmes, peut-être dans le désir d'épouser Ruth, en tout cas dans la volonté de respecter la parole donnée, autre ingrédient d'un « vivre ensemble » harmonieux.

«Vivre ensemble » n'est donc pas simple : le respect des lois et la sensibilité de chacun, la détermination, l'affection, la solidarité, la confiance, l'initiative, l'inventivité, la prise de risque, le respect de la parole donnée, voilà quelques-uns des ingrédients indispensables pour une vie en commun véritablement féconde et épanouissante. Les exemples que la Bible donne montrent majoritairement que cela n'est pas facile : que l'on pense à la difficulté qu'ont les frères à vivre ensemble à cause de la jalousie qu'ils peuvent éprouver les uns envers les autres - Caïn et Abel, Jacob et Ésaü ou encore Joseph et ses frères - ; que l'on pense aussi à la difficulté qu'il y a à vivre avec ceux qui ne nous ressemblent pas, les étrangers, à cause de la peur et du non-respect de l'autre - l'histoire d'Abram que nous avons lue l'illustre bien.

Mais si la Bible souligne l'aspect difficile du « vivre ensemble », elle le fait sans doute pour mettre le lecteur en garde contre certaines impasses qui le guettent dans sa vie et ses relations. Mais à côté de cela, des récits et des lois esquissent des pistes pour un « vivre ensemble » non seulement possible mais aussi enrichissant et vivifiant pour tous. La Loi met en place un cadre dans lequel les relations entre humains seront régulées et qui appelle aussi à des actes concrets de solidarité envers les plus pauvres. Quant à des récits comme celui de Ruth, il montre que la Loi, bien qu'indispensable, n'est pas suffisante. Chacun doit apporter sa pierre à la construction de l'édifice, dans le respect et la reconnaissance mutuels. Alors seulement, il sera possible de dire : "Voici ! Qu'il est bon et qu'il est doux d'être frères aussi ensemble... » (Ps 133).

Elena Di PEDE Faculté de Théologie, Université catholique de Louvain 